\title{
On the Mechanism of the Splay in the Glucose Titration Curve in Advanced Experimental Renal Disease in the Rat*
}

\author{
Stewart W. Shankel, † Alan M. Robson, and Neal S. Bricker $\vdots$ \\ (From the Renal Division, Department of Internal Medicine, Washington University School of \\ Medicine, St. Louis, Mo.)
}

\begin{abstract}
Summary. Glucose titration studies were performed in rats with unilateral chronic pyelonephritis before and after removal of the contralateral control kidneys. Identical studies were performed in animals with unilateral partial renal infarction in which the experimental kidneys had a marked reduction in nephron population but no anatomic deformation in the surviving nephrons. In the initial studies, both groups of animals were free of clinical and chemical abnormalities of uremia. In the follow-up studies uremic abnormalities were present. Minimal splay was observed in the titration curves in the initial studies; marked splay was present in the group data from the same kidneys in the subsequent studies. Thus a marked reduction in the nephron population was associated with the evolution of splay in both groups of animals. In association with the increase in splay, the mean values for maximal glucose transport increased; thus a defect in glucose transport can be excluded as the basis of the splay. Glomerular filtration rate increased proportionately more than the maximal transport of glucose; hence the ratios of glomerular filtration rate to maximal glucose transport increased consistently. The possibility of asymmetric hypertrophy of glomerular and tubular functions among the nephron population imposed by scar tissue or other anatomic deformities was considered, but the results in the animals with partially infarcted kidneys militate against this explanation. The splay also could reflect an asymmetric alteration in the distribution of glomerulotubular balance among the residual units initiated by functional adaptations. Finally, the splay could relate to an alteration in the kinetics of glucose transport without any change in the level of functional homogeneity. The possible nature of these has been considered in the text.
\end{abstract}

\section{Introduction}

The glucose titration curve is believed to provide a sensitive index of nephron homogeneity (1-3).

* Submitted for publication June 27, 1966; accepted October 20, 1966.

Supported by U. S. Public Health Service research grant AM-09976 and graduate training grant T1-AM5248.

$\dagger$ Research Fellow, U. S. Public Health Service.

$\ddagger$ Research Career Award, U. S. Public Health Service.

Address requests for reprints to Dr. Neal S. Bricker, Barnes and Wohl Hospitals, 660 S. Euclid Ave., St. Louis, Mo. 63110.
If all the nephrons in a kidney reabsorb filtered glucose quantitatively until their individual maximal transport rates $(\mathrm{Tm})$ are approached; if the reabsorptive capacity becomes saturated at load/Tm ratios of only slightly greater than unity; if the rate of reabsorption remains constant thereafter despite progressive increments in filtered glucose; and if the level of glomerulotubular balance, with relation to glucose, is the same for all nephrons (i.e., if each nephron reaches $\mathrm{Tm}$ at the same blood sugar level), the nephron population will appear homogeneous and there will be limited splay in the titration curve. 
On the other hand, if any group of nephrons reaches $T m$ either at a lower blood sugar level or at a higher blood sugar level than the predominant population of nephrons, heterogeneity will exist, and there will be splay in the titration curve. Within the framework of this schema, the greater the splay, the greater the functional heterogeneity of the constituent nephrons. However, it is not so well appreciated that splay may also occur in a completely homogeneous population of nephrons due to a uniform alteration in the kinetics of glucose reabsorption (3).

In previous studies from this laboratory, the glucose titration technique was applied to the analysis of functional homogeneity of the diseased kidney of the $\operatorname{dog}(4)$. The experiments were performed on animals with one diseased and one normal kidney. The mean glucose titration curve for the diseased kidneys exhibited minimal splay, and there was no significant difference between the titration curves for diseased and contralateral control organs (4). We interpreted these data to indicate that the pathologic alterations produced by three different types of experimental renal parenchymal disease had not changed the homogeneity of glomerulotubular balance with respect to glucose. More recently, we have performed glucose titration studies on patients with chronic bilateral glomerulonephritis and pyelonephritis (5). In agreement with the data derived from the dog, splay was not increased appreciably in patients whose glomerular filtration rates were reduced by as much as 80 to $85 \%$. However, in contrast to the pattern observed in the dog, splay was increased in patients with GFR values below $15 \mathrm{ml}$ per minute, and when glomerular filtration rate was below $10 \mathrm{ml}$ per minute, the splay was quite marked. The fact that splay did not emerge in the severely diseased kidney of the dog in which there was a normal control kidney and that abnormal splay appeared in human beings only when GFR was greatly reduced suggests that a major reduction in total (i.e., bilateral) functioning nephron population may be implicated in the pathogenesis of splay. The present studies represent an attempt to explore this possibility.

Experiments were performed in the rat. This species was chosen in preference to the dog because the rat is more analogous to man in terms of the ratio of nephrons with short versus long loops of Henle (6). Anatomically the relationship between glomeruli and appended proximal tubules is different in these two populations of nephrons, and conceivably this difference could have relevance in a study of the homogeneity of glomerulotubular balance. In one group of rats, unilateral pyelonephritis was induced and glucose titrations were performed simultaneously on the diseased and control kidneys. Thereafter the control kidney was removed. Approximately 2 weeks after nephrectomy glucose titration studies were repeated. Thus the same nephrons of the same diseased kidneys were studied twice. In the initial studies, with a control kidney in situ, there were a large population of nephrons and relatively normal extracellular fluid composition; in the second set of experiments, the total nephron population was markedly diminished and uremia was present. In another group of animals the nephron population in the experimental kidney was reduced by ligating branches of the renal artery supplying from 50 to $80 \%$ of the renal parenchyma. These kidneys also had a marked reduction in nephron population, but the residual nephrons were free of the pathologic changes observed in the pyelonephritic kidneys. The same experimental sequence described for the animals with pyelonephritis was followed in the rats with unilateral partial renal infarction.

\section{Methods}

Pyelonephritis was induced in the left kidney of female Sprague-Dawley rats of the Holtzman strain. The technique was modified from that previously described in the $\operatorname{dog}(7)$. The exposed kidney was punctured 50 times with a 26-gauge heated cautery needle. An additional 50 punctures were then made with a 25-gauge hypodermic needle that was dipped repeatedly into a culture of Escherichia coli. Efforts were made during this procedure to distribute the punctures uniformly. Bleeding was controlled with pressure. One-half $\mathrm{ml}$ of a 4-hour broth culture of $E$. coli was then administered intravenously via a tail vein, and $4 \mathrm{ml}$ of isotonic saline was injected subcutaneously.

Partial infarction was performed by exposing the left kidney through a midline incision, isolating the main renal artery and its primary and secondary divisions, and then tying the majority of these with a silk ligature. A clear line of demarcation appeared between the ischemic and normal zones of renal parenchyma. The area of infarction could be seen easily.

At the time of the titration studies, the animal was prepared by a modification of the technique described by 
Cotlove (8). Light anesthesia was induced with ether, and the distal part of the left femoral artery was exposed through a small incision. A polyethylene catheter (PE 10) filled with heparinized saline was introduced into the artery and tied firmly into position. The skin incision was closed. A low midline abdominal incision then was made and PE 10 catheter introduced into the right ureter. With the abdomen still open, the bladder was catheterized through the urethra with a PE 90 polyethylene tube in which several side holes had been cut. The bladder catheter was positioned under direct vision and fixed securely in place by suturing it to the skin near the urethral orifice. After urine was seen to flow out of both urethral and ureteral catheters, the abdominal incision was closed. A 25-gauge needle with a polyethylene extension was secured in the tail vein and used for the infusion.

Upon completion of the surgical procedures, $5 \mathrm{ml}$ of isotonic saline was administered subcutaneously, and the animal was placed in a plastic cylinder that was bivalved longitudinally to permit easy positioning of the rat. The lower half of the plastic tube contained two holes through which the rat's hind legs emerged and a third somewhat larger hole for the urethral and bladder catheters. After the animal had been positioned correctly, the two halves of the cylinder were taped together, and the unit was clamped in a horizontal position. The legs were secured to a bar positioned below and at right angles to the cylinder. The tail projected through the open end of the cylinder, and the head was in contact with the atmosphere. The animal was allowed to recover from the anesthetic, and studies were performed in the unanesthetized state. Most of the animals were trained to tolerate confinement in the cylinders before the day of the first experiment.

Glomerular filtration rate was measured with carboxyllabeled inulin $-{ }^{14} \mathrm{C}$. The priming dose of inulin $-{ }^{14} \mathrm{C}$ was administered via the tail vein catheter; the sustaining dose was given through the same catheter by constant infusion. The dose of ${ }^{14} \mathrm{C}$ was calculated to provide a counting rate of approximately $300 \mathrm{cpm}$ in a $10-\mu 1$ sample of plasma. A 1-hour equilibration period was employed in most experiments; however, if the animals had not fully recovered from the anesthetic, if the urine flow was small, if the urine was not glucose-free, or if the animals were not resting quietly in the tube, the equilibration period was prolonged. Five per cent glucose was infused during the equilibration period and the first three clearance periods. After the third period, blood sugar levels were increased in a graduated manner to values in excess of $500 \mathrm{mg}$ per $100 \mathrm{ml}$ by increasing the concentration of glucose in the infusate in a stepwise manner. In general three clearance periods were obtained at each level of glucose infusion, but the duration of each infusion was judged in part by the concentration of glucose in the urine measured semiquantitatively with glucose oxidase paper. The inulin and glucose solutions were contained in separate syringes both of which were placed in the same Harvard constant speed infusion pump (model 600-000). The two solutions mixed at a $\mathrm{Y}$-connection and were de- livered to the rat through the common polyethylene tube. The infusions were delivered at a rate of $60 \mu \mathrm{l}$ per minute $(30 \mu \mathrm{l}$ per minute for glucose and $30 \mu \mathrm{l}$ per minute for inulin).

Individual clearance periods varied from 20 to $30 \mathrm{~min}$ utes in duration depending upon the rate of urine flow. At least 15 clearance periods were obtained during the average experiment. Urine from the individual kidneys was collected in 1-ml graduated tubes. At the end of each collection period pressure was applied over the back of the rat to facilitate emptying of the bladder. This maneuver, after some practice, proved to be a satisfactory means of obtaining complete collections. Arterial blood samples were collected at the beginning, middle, and end of each clearance period in heparinized microhematocrit tubes. These tubes were centrifuged, and the plasma was pooled so as to obtain a plasma glucose concentration for each clearance period approximating an integrated value.

At the completion of an experiment, the rat was reanesthetized, all catheters were removed, and the thigh and abdominal wounds were closed surgically. Immediately thereafter, the right kidney was removed through a posterolateral abdominal incision. Each rat was given 5 $\mathrm{ml}$ of isotonic saline subcutaneously and returned to its cage. During the succeeding days, the animal was weighed daily, and if weight loss and polyuria were excessive, supplementary salt was added to the drinking water. Occasionally the sodium losses in the urine necessitated subcutaneous injection of saline during the first few postoperative days.

The procedure followed during the postnephrectomy experiments was essentially the same as that described above. The femoral artery and tail vein were cannulated again, and in 7 of 9 animals with pyelonephritis and 9 of 12 animals with partial infarction, the ureter draining the experimental kidney was cannulated through an abdominal incision. In the remaining animals, bladder urine was collected so that an additional study could be performed approximately 4 weeks after nephrectomy. The volume of blood collected during each period in the postnephrectomy studies could be reduced because of the low hematocrits in uremic animals, and the rate of infusion of inulin and glucose was reduced by $50 \%$.

For the determination of inulin $-{ }^{14} \mathrm{C}$ in urine, $10-\mu 1$ samples of undiluted urine were pipetted in duplicate into plastic counting vials containing $7 \mathrm{ml}$ of a liquid scintillation solution (9). Plasma was deproteinized by adding $100 \mu 1$ to $1 \mathrm{ml}$ of $2 \%$ perchloric acid. One hundred $\mu 1$ of the supernatant was pipetted in duplicate into the plastic counting vials containing the scintillator. ${ }^{14} \mathrm{C}$ was counted in a Packard Tri-Carb liquid scintillation spectrometer (model 3214), and a total of at least 5,000 counts was obtained on each sample. Plasma samples were found to quench the scintillation system; this was corrected for by recounting the samples after adding a ${ }^{14} \mathrm{C}$ internal standard.

For determination of glucose in urine, 200- $\mu 1$ samples were pipetted into an ion exchange column containing Amberlite 120 and 400 exchange resins, and doubly dis- 
tilled water was used as a wash. The effluent from each column was collected in a graduated cylinder, and water was added to the column until the final volume of effluent equaled $4 \mathrm{ml}$. (Glucose recoveries were found to be complete with this volume of wash.) Glucose was determined on urine filtrates and on deproteinized plasma samples by a glucose oxidase method (10). Glucose titration curves and frequency distribution curves were calculated by the methods described originally by Smith and his associates (1) and recently amplified by Letteri and Wesson (11). The numerical value for maximal glucose transport $\left(T m_{g l u c o s e}\right)$ in each experiment was obtained by averaging all values for glucose reabsorption after the latter had reached a plateau. In general this did not occur until blood sugar levels exceeded $400 \mathrm{mg}$ per $100 \mathrm{ml}$. Some variation in the individual values for glucose reabsorption $\left(T_{\text {glucose }}\right)$ at blood sugar concentrations above the level at which $T m$ occurred accounts for the fact that $T / T m$ values fall above as well as below the line of unity. Individual titration curves were drawn with french curves, and each represents the line that best fits the experimental points. Composite titration curves were drawn according to the method of Smith and co-workers (1). The amount of splay was quantitated by plotting the titration curve on standard graph paper and cutting out the "splay zone" accurately with scissors. (The splay zone represents the area between the titration curve and the theoretical curve of zero splay.) The cut sections of paper were weighed on an analytic balance and expressed in arbitrary "splay units."

\section{Results}

In accordance with a convention previously established the first set of studies in the animals with either unilateral pyelonephritis or unilateral partial infarction and a contralateral uninvolved kidney are referred to as stage II $(7) .^{1}$ The subsequent studies, performed after removal of the control kidney, are referred to as stage III.

A) Animals with unilateral pyelonephritis. Stage II experiments were completed in 16 animals. Nine of these survived and were restudied in stage III, two being studied twice in stage III. At autopsy, in both stage II and stage III rats, the experimental kidneys were contracted, the capsules adherent, and there were multiple scars. On microscopic examination, fibrosis was marked and the scars tended to follow a radial distribution from cortex to medulla. Round cell infiltrates with occasional polymorphonuclear cells appeared in focal areas of the medulla. Hyalinized glomeruli and

\footnotetext{
1 Studies performed in animals with two normal kidneys are designated as stage $I$. However, in the present series of experiments, no studies were performed before the induction of unilateral renal disease.
}

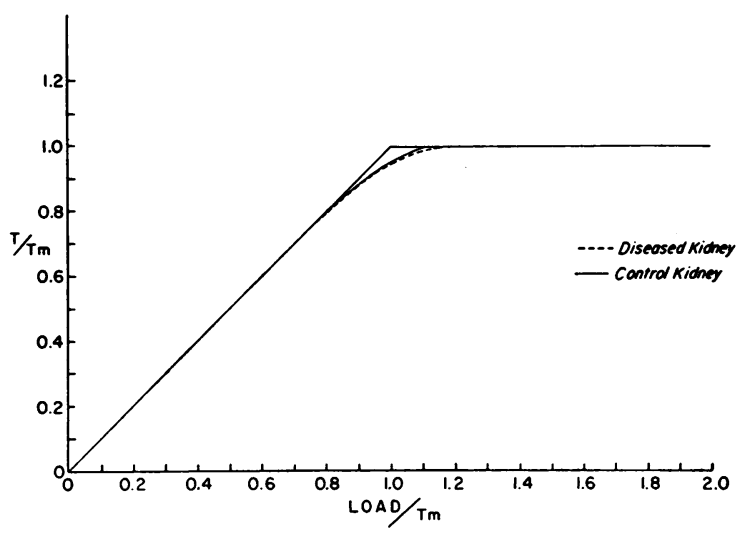

Fig. 1. The mean glucose titration curves for the DISEASED AND CONTRALATERAL CONTROL KIDNEYS IN 16 ANIMALS WITH UNILATERAL PYELONEPHRITIS. $T$ refers to glucose reabsorption in milligrams per minute; load refers to the filtered load of glucose (glomerular filtration rate $\times$ plasma glucose concentration). Both terms are factored by maximal transport ( $\mathrm{Tm}$ ) in order to allow data from both kidneys from different rats to be plotted on the same graph.

atrophic tubules were also present in focal areas. Some involved areas were characterized by the presence of colloid-like material within tubular lumina providing a classical thyroid-like appearance. Uninvolved segments of the renal parenchyma appeared free of pathologic changes.

The stage II studies were performed from 6 to 12 weeks after induction of the lesions. Clinically the rats appeared healthy. Glomerular filtration rate was reduced appreciably in the diseased kidneys, varying from 10 to $43 \%$ of the concurrent value of the contralateral control kidneys. In Figure 1 , the mean titration curves are shown for the diseased and contralateral control kidneys from these animals. One curve is virtually superimposable upon the other. Both leave the theoretical line at a load/Tm value of about 0.8 and return to the line at a load/Tm value between 1.10 and 1.20. Only a minimal amount of splay is present, and the magnitude of the splay differs little between the normal and diseased kidneys. The mean value for the splay zone was $15.5 \mathrm{U}$ for the diseased kidneys and $8.9 \mathrm{U}$ for the control organs. Frequency distribution curves, calculated from the composite titration data, are shown in Figure 2. The graphs for the diseased and contralateral control kidneys are very similar, both demonstrating two peaks analogous to those described by Smith 


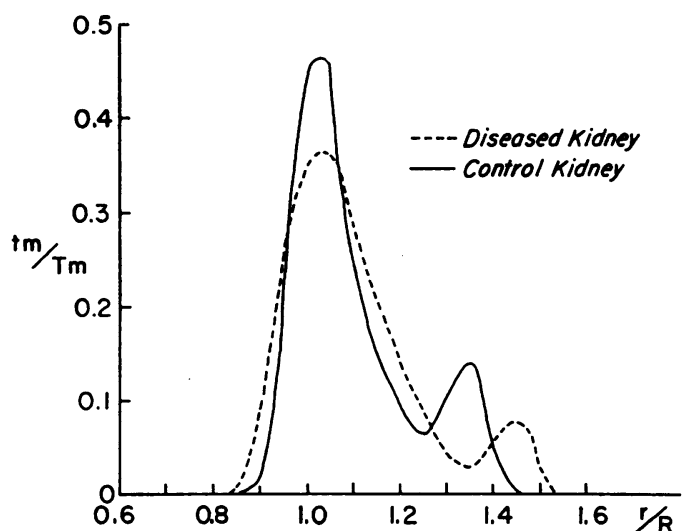

Fig. 2. The Frequency distribution curves for the GLUCOSE TITRATION DATA FROM PYELONEPHRITIC AND CONTROL KIDNEYS FROM THE 16 ANIMALS SHOWN IN FIGURE 1. $\mathrm{tm} / \mathrm{Tm}$ represents the rate of glucose reabsorption in saturated nephrons expressed as a fraction of the $T \mathrm{~m}$ values for the whole kidney considered as a unit; thus this term serves as an index of the fraction of the total nephron population. $r / R$ is an expression for the ratio of glomerulotubular balance of groups of nephrons ( $r$ ) to the mean value for glomerulotubular balance of the whole kidney as a unit ( $R)$. The frequency distribution curve therefore depicts the relative frequency of nephron groups with various levels of glomerulotubular balance related to the mean value for the whole kidney.

and co-workers for normal man (1). Similar curves were observed in previous studies from this laboratory on dogs with unilateral renal disease (4).

At the time of the stage III studies, the animals had lost weight, their coats were dull and lusterless, and despite the reduction in nephrons, polydipsia and polyuria were marked. Blood urea nitrogen levels ranged from 60 to $100 \mathrm{mg}$ per $100 \mathrm{ml}$, and

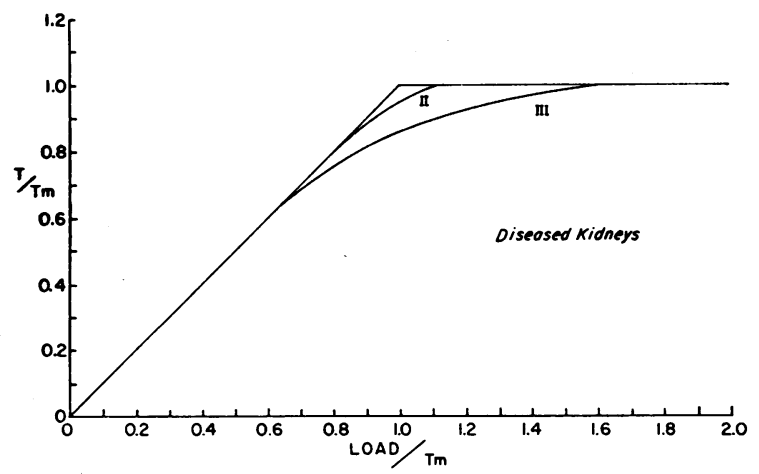

Fig. 3. MEan titration Curves for the diseased KIDNEYS FROM THE NINE ANIMALS STUDIED SEQUENTIALLY IN STAGES II AND III. hematocrits varied from 25 to $35 \%$. In Figure 3 titration curves are shown for the stage III studies and for the stage II studies on the diseased kidneys of the same animals. The transformation is striking. The stage II curve is essentially the same as that obtained for the full group of 16 animals (see Figure 1). On the other hand, the stage III curve leaves the theoretical line at a load/ $\mathrm{Tm}$ value of 0.65 and returns to the line at a load/ $\mathrm{Tm}$ value of 1.60 . For these nine kidneys, the splay in stage II has a mean value of $12.4 \mathrm{U}$, whereas the splay in stage III has a value of 60.4 $U$. The difference is highly significant $(p<$ $0.001)$. A frequency distribution curve was calculated from the composite stage III titration data, and the plot is shown in Figure 4. The curve is remarkably different from that obtained in stage II. In the two animals in which stage III studies were repeated 4 weeks after nephrectomy, the increase in splay observed 2 weeks after nephrectomy persisted.

In Table I, the absolute values for $\mathrm{Tm}_{\text {glucose }}$ and GFR are shown for all stage II and III studies. For the nine animals studied in stage III, the value for $\mathrm{Tm}_{\text {glucose }}$ increased by an average of $15.9 \%$. Glomerular filtration rate in the same animals increased by an average of $45.9 \%$. Thus the ratio of GFR/Tm $\mathrm{m}_{\text {glucose }}$ was greater in stage III than in stage II. In two of the nine animals, absolute values for GFR and $\mathrm{Tm}_{\text {glucose }}$ fell, ${ }^{2}$ yet in both of these animals (as well as the other seven), the ratio of GFR/Tm $\mathrm{m}_{\text {glucose }}$ was greater in stage III than in stage II.

B) Animals with unilateral partial renal infarction. At autopsy the partially infarcted organs typically appeared as small normal kidneys with an appendage of calcified fibrous tissue at one pole. Twelve animals were studied successively in stages II and III, and the composite data are presented in Table II. The splay in the stage II studies was closely comparable in experimental and control kidneys, and as in the animals with unilateral pyelonephritis, one composite titration

2 In one of these the right hind limb became necrotic after the stage II study, and the animal amputated the limb at the pelvis. In the other, a wound abscess developed in the abdominal incision; the animal lost weight and appeared toxic, and the stage III study was performed 10 days rather than 14 days after the stage II study. In both animals it was suspected that the renal disease had progressed between the stage II and III studies. 


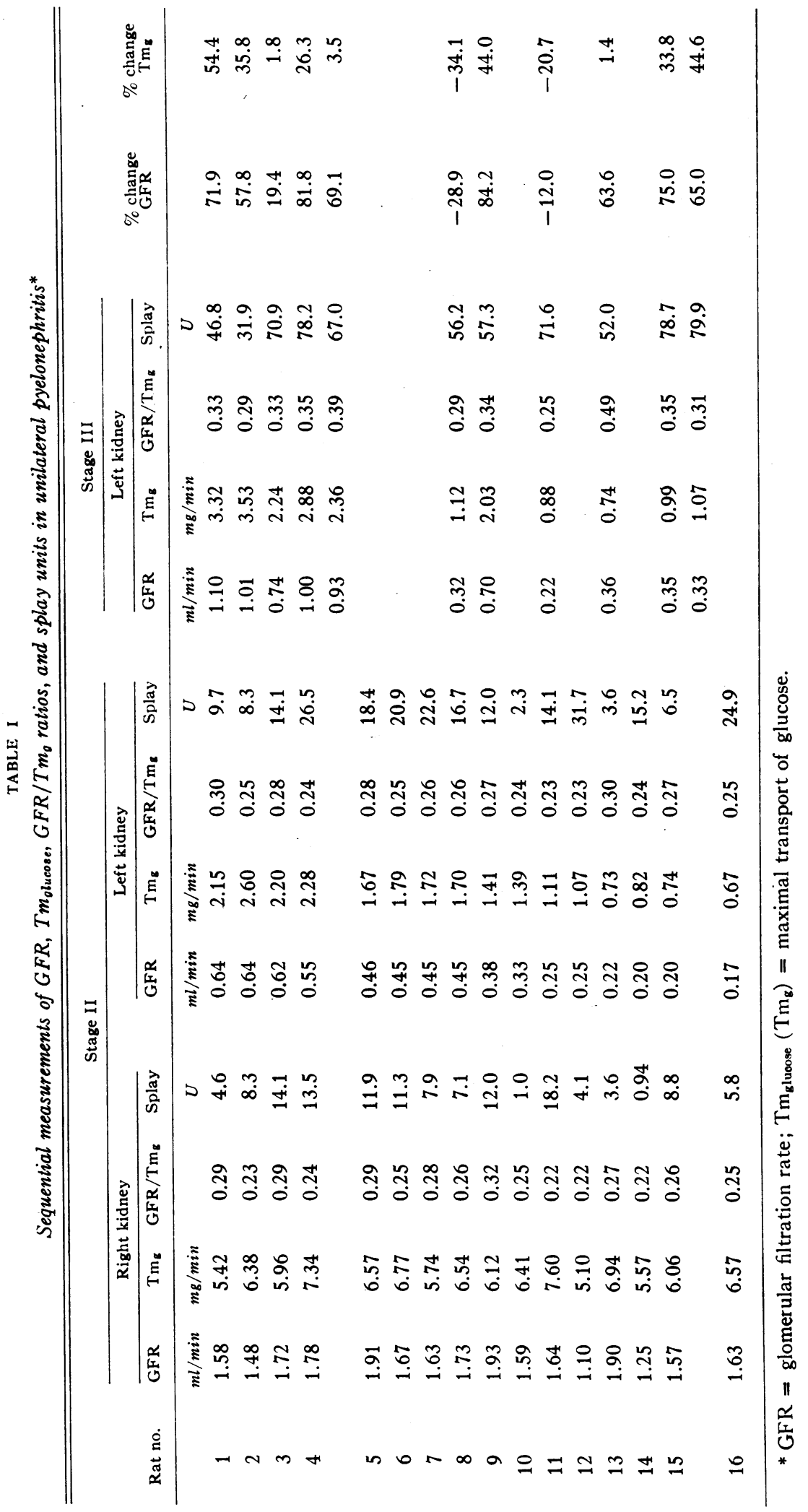




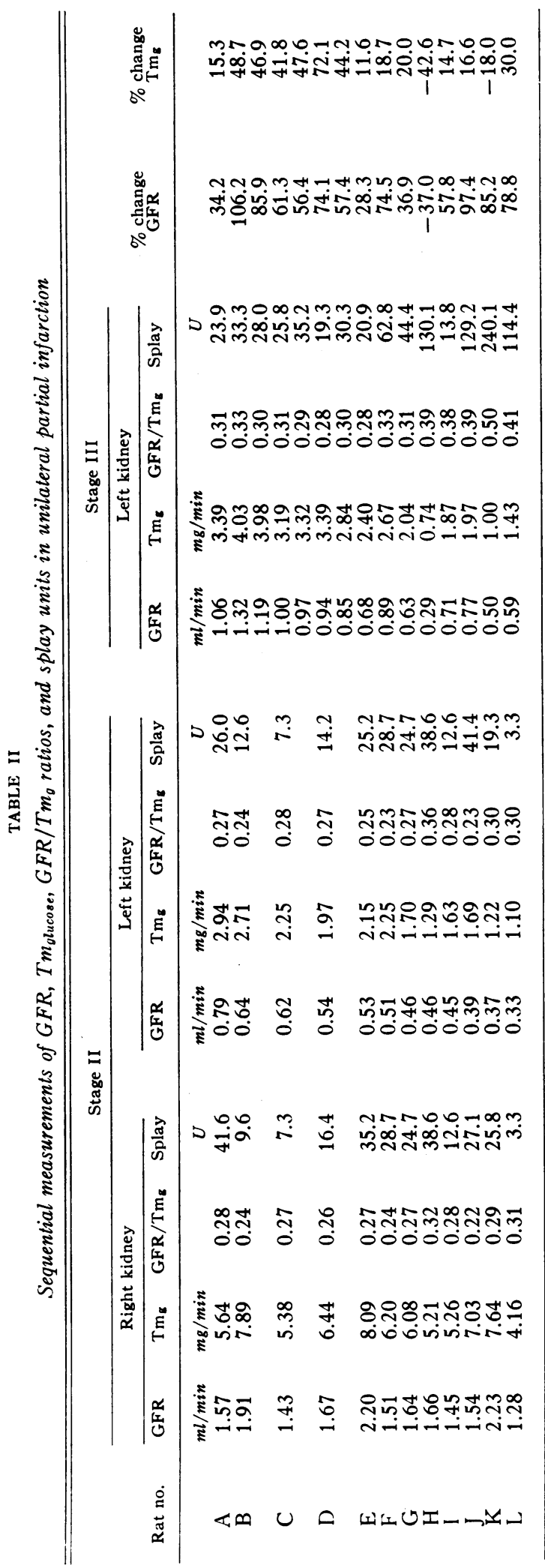

curve was essentially superimposable upon the other (Figure 5). The mean value for splay units was 21.2 for the experimental kidneys and 22.6 for the contralateral control organs. In Figure 6, the composite titration curve for the experimental kidneys in stage III is compared to the stage II curve for the same kidneys. The increase in splay is entirely analogous to that observed in the pyelonephritic animals. The splay units rose from a mean value of 21.2 to 71.5 . The frequency distribution curve for the group showed the same transformation in stage III as was observed in the pyelonephritic animals depicted in Figure 4. In the three animals in which a second stage III study was performed, the splay decreased slightly in one and increased further in two (see Table II).

The absolute values for $\mathrm{Tm}_{\text {glucose }}$ and GFR are included in Table II for the experimental kidneys. $\mathrm{Tm}_{\text {glucose }}$ increased in 10 of the 12 animals; the mean increase for the group was $28.6 \%$. GFR increased in 11 of the 12 animals, and the mean increase was $69.8 \%$. The GFR/Tmglucose ratio increased in all 12 animals between stages II and III.

\section{Discussion}

In the glucose titration studies on patients with chronic pyelonephritis and chronic glomerulonephritis (5) two points of particular interest emerged. The first was that there was little increase in splay in the group of patients with filtration rates between 58 and $15 \mathrm{ml}$ per minute; the second was that there was a marked increase in the splay in patients with filtration rates below $15 \mathrm{ml}$ per minute. In the former group the renal disease was not so severe as in the latter; yet in patients with GFR levels approaching $15 \mathrm{ml}$ per minute (e.g., in the 15 - to 25 -ml-per-minute range), the renal disease and the architectural changes thereof must be classified as moderately to far advanced. The absence of exaggerated splay in this group, therefore, suggested that anatomic deformation of the renal parenchyma does not alone initiate splay in the glucose titration curve. Thus, the change in the titration curve at lower levels of GFR raised the possibility that a critical reduction in nephron population plays a key role in the genesis of the splay phenomenon. The present studies were designed to examine these questions prospectively in 
the experimental animal. The lack of abnormal splay in the pyelonephritic kidneys in the stage II studies supports the view that anatomic alterations per se do not evoke splay in the titration curve. This conclusion also receives support from the previous studies in the dog with severe unilateral renal disease (4). On the other hand, the possibility that a critical reduction in the number of functioning nephrons is important in the genesis of splay is strengthened by the sequential studies performed in stages II and III on the same pyelonephritic kidneys. Furthermore, the emergence of splay between stages II and III in the animals with unilateral partial renal infarction and no anatomic deformation among the functioning nephrons would seem to establish unequivocally a role of nephron loss in the transformation of the glucose titration curve. In this context, the splay in stage III is clearly greater in the rats with the lowest filtration rates and presumably the smallest residual nephron populations (Table II). This is shown graphically in Figure 7, where the stage III titration curves are plotted separately for animals with stage II GFR above $0.5 \mathrm{ml}$ per minute and for those with GFR levels below $0.5 \mathrm{ml}$ per minute. The mean value for splay units in the former group was 31.0 and in the latter group 112.0. In the three animals with GFR below $0.40 \mathrm{ml}$ per minute (this is roughly equivalent to a GFR of $15 \mathrm{ml}$ per minute in an adult human being) the average splay value was $161.2 \mathrm{U}$.

It thus would appear that when the total nephron population is critically reduced, splay evolves in the residual nephrons of the rat whether disease is present or not. The mechanism of the splay, however, still requires clarification. The present studies make unlikely the possibility that the splay can be attributed to a diffuse defect in the maximal reabsorptive capacity for glucose initiated by some consequence of the uremic state, for the average value of $\mathrm{Tm}_{\mathrm{glucose}}$ in the experimental kidneys increased between stages II and III in both pyelonephritic and partially infarcted animals. It may be relevant that the ratio of GFR to $T \mathrm{~m}_{\text {glucose }}$ increased in all animals including those in which absolute values for GFR or $\mathrm{Tm}_{\text {glucose }}$ or both decreased. Thus glomerulotubular balance for glucose consistently changed in the direction of glomerular preponderance. If adaptive changes in filtration rate and glucose reabsorptive

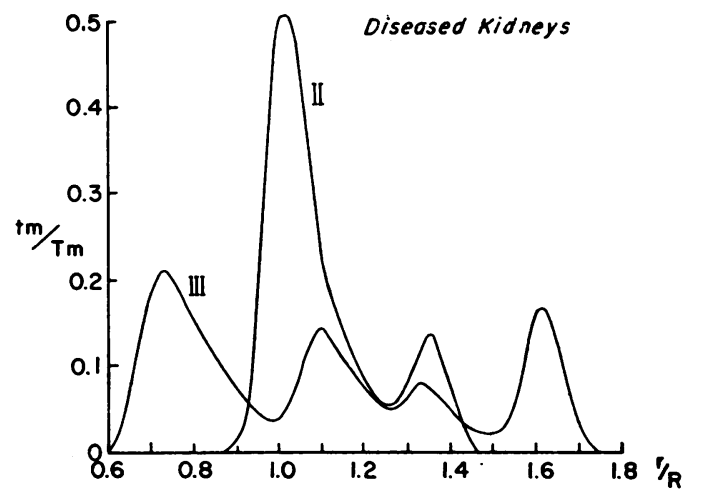

Fig. 4. THE FREQUenCy Distribution CLRVE FOR THE STAGE II versus stage III titration studies. Terms are defined in the legend to Figure 2.

capacity among the nephrons occurred in an asymmetric fashion, the urine-forming nephrons could be converted into a heterogeneous group, and the splay then would truly reflect the emergence of functional heterogeneity. Such asymmetric hypertrophy might correspond to an asymmetric distribution of pathologic changes particularly of scar tissue in the pyelonephritic kidneys. The data in the animals with partially infarcted kidneys do not exclude the possibility that splay in the pyelonephritic kidneys relates to scar tissue, but they make it less likely. It is possible, however, that functional adaptations might alter the distribution of glomerulotubular balance with respect to glucose in the absence of pathologic changes. For example, a disproportionate increase in blood flow and filtration rate in juxtamedullary nephrons could contribute to functional heterogeneity and thus to splay in the titration curve. As alluded to

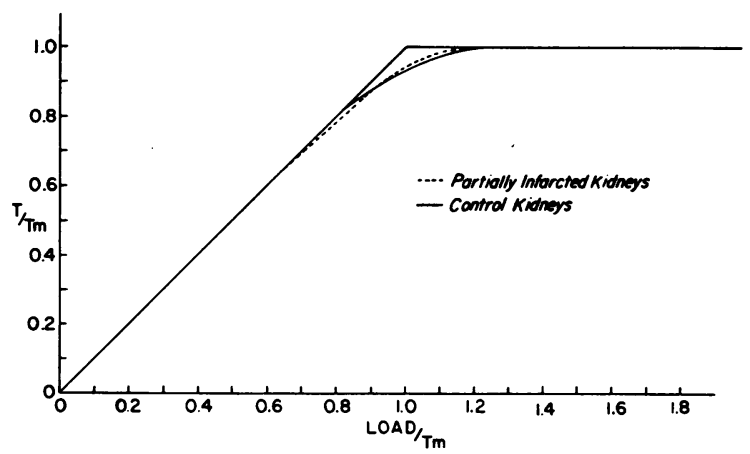

Fig. 5. The MeAN glucose titration CURVES for the DISEASED AND CONTRALATERAL CONTROL KIDNEYS IN 12 ANIMALS WITH PARTIAL RENAL INFARCTION AND CONTRALATERAL CONTROL KIDNEYS. 


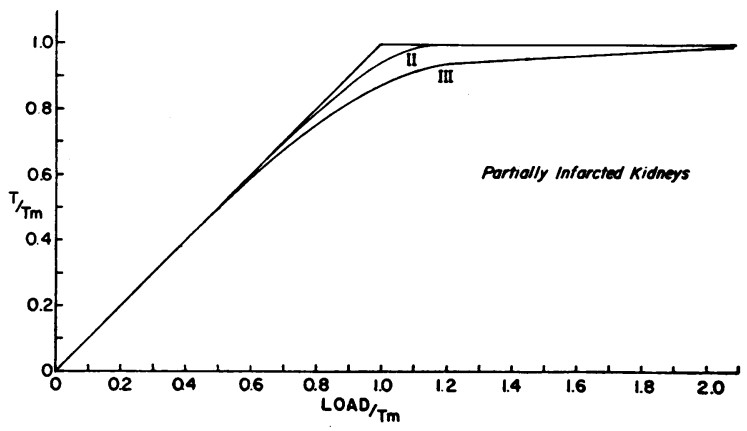

Fig. 6. MEAN TITRATION CURVES FOR THE EXPERIMENTAL KIDNEYS WITH PARTIAL RENAL INFARCTION FROM THE 12 ANIMALS STUdied SEQUENTIALLY IN STAGES II AND III.

earlier, it is conceivable that glomerulotubular balance is different in the juxtamedullary nephrons than in the cortical units. The final possibility is that the splay has a kinetic explanation and does not necessarily connote a change in functional homogeneity. There appears to be an increase in GFR per nephron when the nephron population is reduced (Tables I and II, reference 4 ), and there is characteristically a reduction in fractional reabsorption of sodium and water in advanced renal disease. In micropuncture studies in the rat with experimental pyelonephritis, the disease is associated with a reduction in fractional reabsorption in the proximal tubule (12). Both the increase in GFR and the decrease in fractional reabsorption would increase the volume of intratubular fluid in the segments of the nephron responsible for glucose reabsorption; velocity of flow may also be increased, and the kinetics of glucose reabsorption could well be altered in a

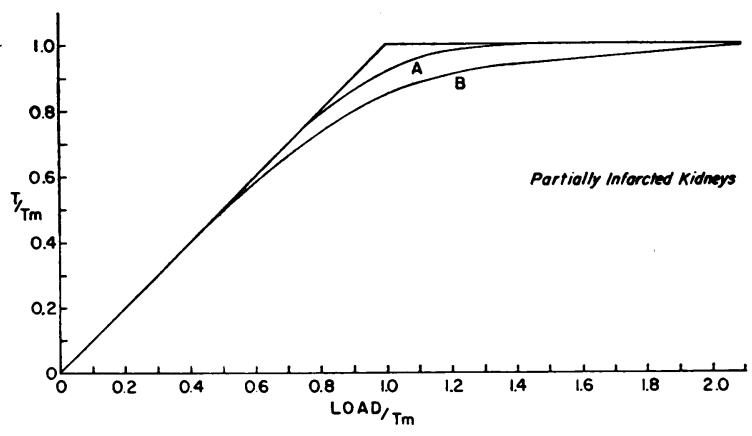

Fig. 7. MEAN titration CURVES FOR the Partially INFARCTED KIDNEYS WITH GLOMERULAR FILTRATION LEVELS IN STAGe II ABove 0.5 ML PER MinUte (A) AND Below 0.5 MIL PER MINUTE (B). uniform fashion throughout the nephron population in the absence of either heterogeneity or impairment of maximal velocity of glucose transport. Additional explanations for kinetic alterations have been considered separately (5).

\section{References}

1. Smith, H. W., W. Goldring, H. Chasis, H. A. Ranges, and S. E. Bradley. The William Henry Welch Lectures. II. The application of saturation methods to the study of glomerular and tubular function in the human kidney. J. Mt Sinai Hosp. 1943, 10, 59.

2. Bradley, S. E., J. H. Laragh, H. O. Wheeler, M. MacDowell, and J. Oliver. Correlation of structure and function in the handling of glucose by the nephrons of the canine kidney. J. clin. Invest. 1961, 40, 1113.

3. Berliner, R. W. Outline of renal physiology in Diseases of the Kidney, M. B. Strauss and L. G. Welt, Eds. Boston, Little, Brown, 1963, p. 30.

4. Bricker, N. S., T. Orlowski, S. W. Kime, Jr., and P. A. F. Morrin. Observations on the functional homogeneity of the nephron population in the chronically diseased kidney of the dog. J. clin. Invest. 1960, 39, 1771.

5. Rieselbach, R. E., S. W. Shankel, E. Slatopolsky, H. Lubowitz, and N. S. Bricker. Glucose titration studies in patients with chronic progressive renal disease. J. clin. Invest. 1967, 46, 157.

6. Sperber, I. Studies on the mammalian kidney. Zoologische Beitrage Aus Uppsala 1944, 22, 249.

7. Bricker, N. S., S. Klahr, and R. E. Rieselbach. The functional adaptation of the diseased kidney. I. Glomerular filtration rate. J. clin. Invest. 1964, 43, 1915.

8. Cotlove, E. Simple tail vein infusion method for renal clearance measurements in the rat. J. appl. Physiol. 1961, 16, 764.

9. Bray, G. A. A simple efficient liquid scintillator for counting aqueous solutions in a liquid scintillation counter. Analyt. Biochem. 1960, 1, 279.

10. Bergmeyer, H. U., and E. Bernt. D-Glucose determination with glucose oxidase and peroxidase in Methods of Enzymatic Analysis, 1st ed., H. U. Bergmeyer, Ed. New York, Academic Press, 1963, p. 123.

11. Letteri, J. M., and L. G. Wesson, Jr. Glucose titration curves as an estimate of intrarenal distribution of glomerular filtrate in patients with congestive heart failure. J. Lab. clin. Med. 1965, 65, 387.

12. Lubowitz, H., M. Purkerson, and N. S. Bricker. Micropuncture studies on individual nephrons in the rat with chronic pyelonephritis. J. clin. Invest. $1965,44,1071$. 